\title{
ANÁLISE TEMPORAL DAS TAXAS DE SUICÍDIOS NO ESTADO DE MINAS GERAIS COM MODELOS ARIMA ${ }^{1}$
}

\author{
Rodnei Alves Marques ${ }^{2}$ \\ Spencer Barbosa da Silva
}

\begin{abstract}
RESUMO
O autoextermínio tem aumentado em Minas Gerais em todas as faixas etárias. Tal fato, além de resultar em perda de vidas humanas, demanda recursos públicos que poderiam ser aplicados em outras áreas da saúde. Isto posto, esse trabalho tem como objetivo modelar e fazer previsões para as taxas específicas de suicídio por sexo no estado de Minas Gerais. Estas análises, juntamente com estudos clínicos, podem compor mais uma alternativa para os órgãos de saúde, auxiliando-os no preparo de ações que possam diminuir de forma efetiva o número de suicídios. As séries foram ajustadas com a metodologia de Box \& Jenkins com utilização dos softwares R e GRETL. Foi observada uma tendência crescente histórica nas séries ao longo dos anos para as taxas específicas de suicídios masculinos e femininos e conclui-se que este crescimento ainda prevalece para os próximos anos caso não haja nenhuma intervenção mais efetiva.
\end{abstract}

Palavras-chave: Séries temporais. Taxa de suicídios. Modelos ARIMA. Previsões.

\section{TEMPORAL ANALYSIS OF SUICIDE RATES IN THE STATE OF MINAS GERAIS WITH ARIMA MODELS}

\begin{abstract}
Self-extermination has increased in Minas Gerais in all age groups. This fact, besides resulting in the loss of human lives, demands public resources that could be applied in other areas of public health. From this point, this work aims to model and make predictions for specific suicide rates by gender in the state of Minas Gerais. These analyses, combined with clinical studies, can be another alternative for health public agencies, helping them to prepare actions that can effectively reduce the number of suicides. The series were adjusted using the Box \& Jenkins methodology using the R and GRETL softwares.A historical growing trend was observed in the series over the years for specific rates of male and female suicides and we concluded that this growth shall remain for the next years if there is no other effective intervention.
\end{abstract}

Keywords: Time series. Suicide rate. ARIMA Models. Forecasts.

1 Como citar este artigo:

MARQUES, R. A.; SILVA, S. B. Análise temporal das taxas de suicídios no estado de Minas Gerais com modelos ARIMA. ForScience, Formiga, v. 8, n. 2, e00842, jun./dez. 2020. DOI: $10.29069 /$ forscience.2020v8n2.e842.

2 Autor para correspondência: Rodnei Alves Marques, rodnei.marques@ifmg.edu.br 


\section{INTRODUÇÃO}

As ocorrências de suicídios têm se tornado cada vez mais frequentes no Brasil e no mundo, com crescimento progressivo nos últimos anos. De acordo com a Organização Mundial da Saúde (OMS), para cada adulto que morreu por suicídio, pode ter havido mais vinte outros tentando suicidar-se. $\mathrm{O}$ ato suicidio é considerado a terceira maior causa de óbitos por fatores externos, perdendo apenas para homicídios e acidentes de trânsitos, nesta ordem. Enquadram-se como suicídios atos que causam a própria morte de forma intencional.

De acordo com Filho e Zerbini (2016), os índices de suicídios aumentaram em todas as faixas etárias, mas tentativas de suicídios são maiores entre as mulheres e jovens, sendo a intoxicação por medicamentos o método mais utilizado. No entanto, homens brasileiros provocam autoextermínio efetivo em maior intensidade, ou seja, a população masculina utiliza métodos de maior letalidade. Segundo os autores, os métodos mais utilizados pelos homens são enforcamentos e armas de fogo.

Resultados semelhantes foram encontrados por Ribeiro et al. (2018). Os autores analisaram 169 tentativas de suicídios na cidade de Uberaba em Minas Gerais e concluíram que, nas tentativas de suicídio por pessoa do sexo feminino, destacaram-se o uso de medicamentos e envenenamento e, para homens, os principais meios de auto agressão foram enforcamentos, seguido de auto-intoxicação. Para as análises da série temporal, os autores utilizaram análises descritivas e regressão polinomial.

No trabalho realizado por Gonçalves, Gonçalves e Júnior (2011), os autores abordam a dependência espacial do número de casos, ou seja, a constatação do "efeito contágio" espacial a partir de amostras de microrregiões geográficas brasileiras. Eles defendem a criação de um plano nacional de prevenção ao suicídio, que objetive sensibilizar a sociedade e mostrar que o suicídio não está limitado ao indivíduo, mas sim relacionado à sociedade como um todo.

Do ponto de vista econômico, o suicídio e suas tentativas representam enorme custo para a sociedade. Primeiro, porque demanda recursos públicos que poderiam ser aplicados em outras áreas da saúde, e, segundo, por que resulta na perda do capital humano. Em uma pesquisa realizada pelo Instituto de Pesquisas Econômicas Aplicadas - (IPEA, 2020), o valor médio das internações registradas por tentativas de suicídios entre os anos de 1998 e 2004 foi de R \$507,00 (quinhentos e sete reais), com média de internação de quatro dias.

Analogamente, ainda considerando o ponto de vista econômico, Branas et al. (2015) utilizaram os modelos ARIMA para analisar as séries temporais de suicídios mensais na Grécia entre os anos de 1983 e 2012. Os autores alertam sobre o aumento da taxa de suicídios no mesmo período em que medidas de austeridade econômica são aplicadas à população. As análises mostraram que as medidas de austeridade econômica aplicadas em janeiro de 2002, outubro 
de 2008, junho de 2011 e abril de 2012 provocaram aumentos significativos nas ocorrências de suicídios entre a população.

Ademais, a prevalência de autoextermínio entre os homens condiciona um valor total de custo 1,66 vezes maior que o valor gasto para as internações de mulheres, alertam Silveira, Santos e Ferreira (2012). A média de permanência e valor médio de internações do sexo masculino são maiores, o que denota um quadro de maior gravidade. Porém as internações do sexo feminino são mais dispendiosas, requerendo mais gastos com equipamentos e um maior valor agregado por dia.

Diante deste tema, o objetivo deste trabalho é modelar e fazer previsões para as taxas específicas de suicídios no estado de Minas Gerais, de modo que as análises sejam mais uma alternativa às secretarias de saúde, conselhos regionais de psicologia e também organizações não governamentais prepararem, juntamente com estudos clínicos, no planejamento de ações que possam reduzir de forma efetiva as taxas de suicídios no estado. Os dados de suicídios por sexo foram obtidas nos bancos de dados do Instituto de Pesquisas Econômicas Aplicadas (IPEA) e do Instituto Brasileiro de Geografia e Estatística (IBGE).

Para ajuste dos modelos foi utilizada a metodologia de Box \& Jenkins, que consiste nos modelos autorregressivos integrados de médias móveis às séries das taxas de suicídios em Minas Gerais. Essa metodologia parte do princípio de que os modelos temporais podem ser desenvolvidos em ciclos interativos, no qual a estrutura do modelo é baseada nos próprios dados. Foram escolhidos os modelos que apresentaram melhores ajustes e feitas previsões para os anos de 2019, 2020 e 2021.

As séries foram analisadas no software R e no software GRETL. O artigo está organizado da seguinte forma: na seção 2 é apresentada um revisão de literatura com citações de trabalhos que desenvolveram previsões com modelos ARIMA; na seção 3 são abordados os materiais e métodos com apresentação dos dados e a metodologia adotada; na seção 4 os resultados e discussões com apresentação dos melhores modelos, consolidados com testes estatísticos; por fim, na seção 5, são apresentados os resultados e discussões.

\section{REVISÃO DE LITERATURA}

Uma série temporal pode ser definida como um conjunto de observações discretas ou contínuas ao longo do tempo, que apresentam dependência de um instante para outro. Estas observações podem ser decompostas nos componentes de tendência, ciclo e sazonalidade. As análises e previsões podem ser feitas no domínio temporal com modelos paramétricos ou no domínio de frequências com modelos não paramétricos.

O conceito de fazer previsões é antigo e pode ser encontrado nas mais diversas áreas 
do conhecimento, como por exemplo: engenharia, economia, saúde, meio ambiente, etc. Com previsões futuras é possível tomar decisões prévias e fazer correções em uma sequência de eventos. O pressuposto básico da análise de séries temporais é a de que fatores que influenciam o comportamento da série no passado continuarão a fazê-lo no futuro, conforme comentam Araujo et al. (2009).

Com a análise de uma série temporal podemos investigar o mecanismo gerador da série, fazer previsões, descrever o comportamento e procurar periodicidades pertinentes. Então, a notação utilizada nesse trabalho para denotar uma série temporal de tamanho $n$, será:

$$
Z\left(t_{1}\right), Z\left(t_{2}\right), Z\left(t_{3}\right), \ldots, Z\left(t_{n}\right)
$$

em que $Z_{j}$ é a componente da série no tempo $j$.

De acordo com Morettin e Toloi (2006), uma das suposições mais frequentes que se faz a respeito de uma série temporal é sobre sua estacionalidade, ou seja, se o seu comportamento, ao longo do tempo, se desenvolve aleatoriamente ao redor de uma média. A maneira mais tradicional de analisar uma série é através da sua decomposição nas componentes de tendência, ciclo e sazonalidade.

A tendência de uma série indica se ela cresce, decresce ou permanece estável e qual a velocidade destas mudanças. De acordo com Campos (2008), tendência em uma série temporal é a mudança gradual observada por meio da variação dos seus valores ao longo do tempo e que se mantém ao se moverem os componentes de ciclos, sazonalidade e fatores aleatórios.

As sazonalidades referem-se a movimentos de padrões regulares ou repetidos de curto prazo em torno de uma linha de tendência, em que os dados da série temporal parecem obedecer a períodos como dias, semanas, meses, etc. São classificados como movimentos sistemáticos causados por fenômenos não econômicos e aparecem quando as observações são intra-anuais, (MORETTIN; TOLOI, 2006).

\subsection{Modelos ARIMA}

Uma série temporal é um conjunto de observações feitas e armazenadas ao longo do tempo que possuem tendência serial. São observações que podem ser contínuas no tempo ou discretas ou aquelas que são feitas em um intervalo de tempo específico. Os modelos utilizados para descrever séries temporais são processos controlados por leis probabilísticas chamados processo estocástico, definido abaixo, (MORETTIN; TOLOI, 2006).

Definição 1: Seja $T$ um conjunto arbitrário. Um processo estocástico é uma família $\{Z=$ $Z(t), t \in T\}$, tal que, para cada $t \in T, Z(t)$ é uma variável aleatória. 
O modelo ARIMA, também conhecido como metodologia Box \& Jenkins, é composto por um processo autorregressivo (AR) que utiliza termos defasados da série temporal como variável explicativa. Após aplicação de testes, se houver indicação do uso de variável defasada uma vez, o modelo é chamado de autorregressivo de primeira ordem, defasada duas vezes, de segunda ordem, e assim por diante, (GUJARATI; PORTER, 2011).

O modelo tem como princípio que a série temporal seja gerada por um processo estocástico cuja natureza pode ser representada através de um modelo. A notação empregada para designação do modelo é $\operatorname{ARIMA}(p, d, q)$, em que $p$ representa o número de parâmetros autorregressivos, $d$ o número de diferenciações para que a série se torne estacionária e $q$ o número de parâmetros de médias móveis, ou seja, uma combinação linear dos termos que ocorrem atualmente e em momentos do passado.

Em situações em que a série a ser analisada não apresente estacionalidade, aplicamse diferenças até que a nova variável seja estacionária; se a série indicar estacionalidade após aplicação da primeira diferença, então a série é integrada de ordem $d=1$. Em caso negativo, aplica-se uma segunda diferença à nova variável e a série passa a ser de ordem $d=2$, e assim por diante.

Definição 2: Um processo $Y_{t}$, quando estacionário, é conhecido como auto-regressivo de ordem p, se:

$$
Y_{t}=\mu+\sum_{i=1}^{p} \phi_{i} Y_{t-i}+\varepsilon_{t}
$$

em que $\varepsilon_{t} \sim N\left(0, \sigma_{\varepsilon_{t}}^{2}\right)$.

O modelo $\mathrm{MA}(q)$ assume que a série é gerada através de uma combinação linear de $q$ sinais de ruídos $\varepsilon_{t-1}$, aleatórios e independentes entre si, definido como:

Definição 3: Um processo $Y_{t}$, quando estacionário, é conhecido como médias móveis de ordem q, se:

$$
Y_{t}=-\sum_{i=0}^{q} \theta_{i} \varepsilon_{t-i}
$$

em que $\varepsilon_{t} \sim N\left(0, \sigma_{\varepsilon_{t}}^{2}\right)$.

A combinação dos modelos $\operatorname{AR}(p)$ e $\mathrm{MA}(q)$, quando o processo é estacionário, dá ori- 
gem ao modelo $\operatorname{ARMA}(p, q)$, no qual

$$
Y_{t}=\mu+\sum_{i=1}^{p} \phi_{i} Y_{t-i}-\sum_{i=0}^{q} \theta_{i} \varepsilon_{t-i}
$$

em que $\varepsilon_{t} \sim N\left(0, \sigma_{\varepsilon_{t}}^{2}\right)$.

Com combinações do modelo $\operatorname{AR}$ com MA, chamado modelo $\operatorname{ARMA}(p, q)$, obtémse representações mais relevantes com um número menor de parâmetros. Na proposta para modelos não estacionários, com os modelos ARIMA $(p, d, q)$, temos uma série com valores finitos para realizar as diferenças $d$, para tornar a série estacionária, representado por $W_{t}=\nabla^{d} Y_{t}$.

\subsection{Etapas da modelagem}

Na aplicação dos modelos de Box \& Jenkins, com objetivo de fazer previsões em séries temporais, a primeira fase consiste em identificar o processo aleatório gerador dos dados para, em seguida, estimar os parâmetros que o caracterizam e verificar se as hipóteses do modelo são cumpridas. Caso contrário, uma nova fase de identificação deve ser considerada até que a verificação das hipóteses seja finalmente satisfatória, permitindo assim, fazer previsões.

\section{- Identificação}

Na identificação do modelo ARIMA, são empregadas técnicas que possam identificar a estrutura do modelo de forma a permitir a identificação dos valores de $p, d$ e $q$, que caracterizam o processo estocástico. Primeiramente são feitas diferenciações na série original, quantas forem necessárias, para que a série resultante seja estacionária. O número de diferenciação $d$ é aquele para que a função de autocorrelação amostral da série transformada decresça rapidamente para zero.

Para apontar o processo $\operatorname{AR}(p)$, é feita análise da função de autocorrelação parcial ( facp) e, para o processo MA $(q)$, é feita uma análise da função de autocorrelação ( $f a c)$, para determinação dos parâmetros $p$ e $q$, (MORETTIN; TOLOI, 2006). Vale salientar que deve-se usar um modelo com melhor ajuste e menor número de parâmetros, chamado princípio da parcimônia. Em outras palavras, dados dois modelos com ajustes iguais, é escolhido aquele com menor número de parâmetros.

\section{- Estimação dos parâmetros}

Esta etapa é executada por meio de software específico para análise de séries temporais. São diversos métodos para estimação dos parâmetros com resultados semelhantes, embora 
exista diferença quanto à eficiência computacional. De modo mais geral, utiliza-se algum procedimento interativo de estimação de mínimos quadrados não linear ou utilizam-se métodos de máxima verossimilhança, (MORETTIN; TOLOI, 2006).

\section{- Verificação}

Para que o uso do modelo seja eficaz na predição da variável dependente em estudo, o pesquisador necessita examinar previamente os pressupostos da regressão, bem como identificar as consequências da sua violação. Entre os pressupostos estão: normalidade, independência e média dos resíduos.

Ao ajustar um modelo ARIMA, o objetivo é capturar toda estrutura de dependência presente na série. Então, os resíduos do modelo não devem apresentar nenhum tipo de dependência no tempo. Em um modelo ARIMA ajustado adequadamente, os erros de previsão (resíduos) devem ser uma sequência de ruídos brancos, ou seja, não devem existir auto-correlações e autocorrelações parciais significativas. Os testes estatísticos nessa etapa envolvem verificações da condição de estacionalidade, normalidade dos resíduos, análise de significância dos parâmetros estimados e critérios de seleção de modelo. Os testes utilizados para verificação foram: Teste de Box \& Pierce, Critério de Akaike e o teste de Shapiro-wilk.

\section{- Previsões}

Um dos principais objetivos da análise de séries temporais é prever um valor $Z_{t+h}, h \geq 1$, supondo que temos as observações ..., $Z_{t-2}, Z_{t-1}, Z_{t}$, até o instante $t$, que é chamado origem das previsões. A notação mais utilizada é denotada como $\hat{Z}_{t}(h)$, ou seja, previsão de origem $t$ e horizonte $h$.

Essas previsões também podem ser obtidas por meio de intervalos de confiança para os $Z_{t+1}, Z_{t+2}$, etc. No entanto, a variância aumenta com $h, \operatorname{logo}$ as amplitudes desdes intervalos aumentam à medida que se afasta da origem $t$, caracterizando o aumento da incerteza das previsões à medida que $h$ cresce.

Nesse trabalho foi utilizada uma técnica para avaliar as previsões, que consiste na retirada dos últimos valores reais da série e fazer o ajuste com ausência desses valores, para obter previsões e compará-las com os valores reais. Verificando assim se esses valores estão dentro do intervalo de confiança gerados pela previsão. 


\section{METODOLOGIA}

Os dados dos números de suicídios por sexo no estado de Minas Gerais, entre os anos de 1992 e 2018, foram obtidos no banco de dados do Instituto de Pesquisas Econômicas Aplicadas (IPEA), vinculado ao Ministério da Economia. Os trabalhos do IPEA estão disponíveis gratuitamente por meio de publicações, seminários e um canal de TV. Os dados relativos à população por sexo do estado de Minas Gerais, também entre os anos 1992 e 2018, foram obtidos por meio de estimativas feitas pelo Instituto Brasileiro de Geografia e Estatística (IBGE). O IBGE constitui o principal provedor de dados e informações do país, que atende às necessidades dos mais diversos segmentos da sociedade civil.

Para padronizar os dados por sexo foi utilizada a taxa específica de suicídios (TES) por dez mil habitantes, de acordo com a equação (5):

$$
{ }_{n} T E S_{x}=\frac{{ }_{n} S_{x}}{{ }_{n} P_{x}} \cdot 10.000,
$$

em que ${ }_{n} S_{x}$ é o número de suicídios por sexo e ${ }_{n} P_{x}$ a população por sexo no estado de Minas Gerais.

Inicialmente, foi considerada uma análise descritiva das séries com a construção dos gráficos, para observar indícios de tendência e sazonalidade. Verificou-se a necessidade de transformação dos dados, por meio do gráfico amplitude versus média.

Foram aplicados os testes de Dickey Fuller Aumentado (ADF) e Kruskall-Wallis para tendência e sazonalidade, em seguida analisadas as funções de autocorrelação $(f a c)$ e autocorrelação parcial ( $f a c p$ ) na série livre de tendência para estimar a ordem do modelo. Após o ajuste dos modelos adequados, foram analisadas as funções fac e facp dos resíduos e aplicado o teste de Ljung-Box para verificar se os resíduos são ruído branco.

A princípio foram retiradas as taxas específicas de suicídios dos anos 2016, 2017 e 2018 e feitas previsões com os melhores modelos ajustados, escolhido de acordo com o critério de Akaike (AIC), para esses anos. Com objetivo de fazer a comparação das taxas específicas reais com os valores previstos pelo modelo melhor ajustado. Após essa análise preliminar, foi incluído novamente às séries os valores retirados, ajustado novamente os modelos e feitas previsões para os anos 2019, 2020 e 2021. Por fim, um diagnóstico gráfico do modelo ajustado.

As análises foram feitas com utilização do software R (Core Team, 2018), com emprego dos pacotes de ferramentas forecast - (HYNDMAN; KHANDAKAR, 2008), urca - (PFAFF, 2008), FitAR - (MCLEOD; ZHANG, 2008), runstats - (KARAS; URBANEK, 2019), randtests - (CAEIRO; MATEUS, 2014) e lmtest - (ZEILEIS; HOTHORN, 2002). Também foi utilizado o software GRETL, (ANDRADE, 2013). 


\section{RESULTADOS E DISCUSSÃO}

São apresentados os modelos de ajustes obtidos com aplicação da metodologia de Box et al. (1994) nas séries das taxas específicas de suicídios por sexo no estado de Minas Gerais, com objetivo de fazer previsões para que ações sociais possam ser direcionadas a essa temática.

\subsection{Identificação dos modelos}

Nesta proposta foram trabalhados os dados da taxa específica de suicídios na população masculina e feminina no estado de Minas gerais entre os anos de 1992 e 2018, obtidos no banco de dados do Instituto de Pesquisas Econômicas Aplicadas (IPEA), vinculado ao Ministério da Economia do governo federal. Na Figura 1, são mostradas as séries anuais de suicídios da taxa específica para a população feminina (em vermelho) e para população masculina (em azul) no estado de Minas Gerais no período estudado.

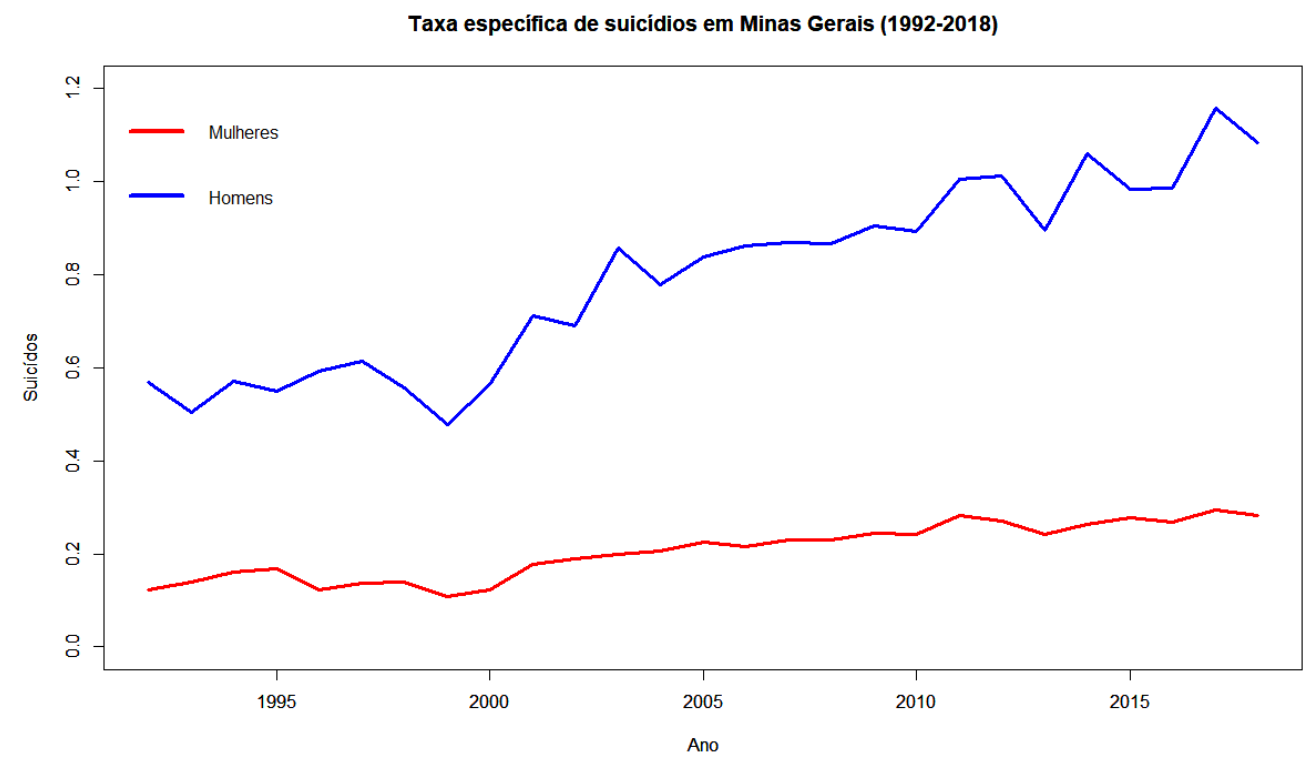

Figura 1 - Séries de suicídios por sexo no estado de Minas Gerais (1992-2018)

A análise da série em estudo foi iniciada a partir de uma inspeção visual do seu gráfico, Figura 1, que, a princípio, mostra indícios da presença de tendência ao longo do tempo. Percebese também as informações observadas por Filho e Zerbini (2016) e Ribeiro et al. (2018), de que, apesar das tentativas de suicídios serem mais significativas entre as mulheres, os homens provocam autoextermínio de forma mais efetiva.

Para verificar a necessidade de transformação dos dados, foi feito o gráfico amplitude 
versus média, constatou-se a não necessidade de transformação dos dados, já que a média não se mostrou diretamente proporcional à amplitude.

Para análise de série temporal é necessário que ela seja estacionária, pois séries não estacionárias podem fornecer resultados preditivos viesados. Portanto, as séries foram submetidas ao teste de raiz unitária Dickey Fuller aumentado (ADF) para verificar a existência ou não de estacionalidade em nível. Com p-valores de 0,2954 para a série feminina e 0,0763 para a série masculina, mostra-se que as séries não são estacionárias, ou seja, o número de suicídios por 10.000 habitantes tem aumentado, ao longo dos anos, para as duas populações (feminina e masculina).

Para transformar uma série não estacionária em estacionária, aplica-se a ela diferenças até que a série apresente estacionalidade. O número de diferenças necessárias para transformála em estacionária será a componente de integração $(d)$ do modelo $\operatorname{ARIMA}(p, d, q)$. Após a aplicação da primeira diferença, percebe-se, pelo gráfico na Figura 2, que as séries diferenciadas apresentam estacionalidade.
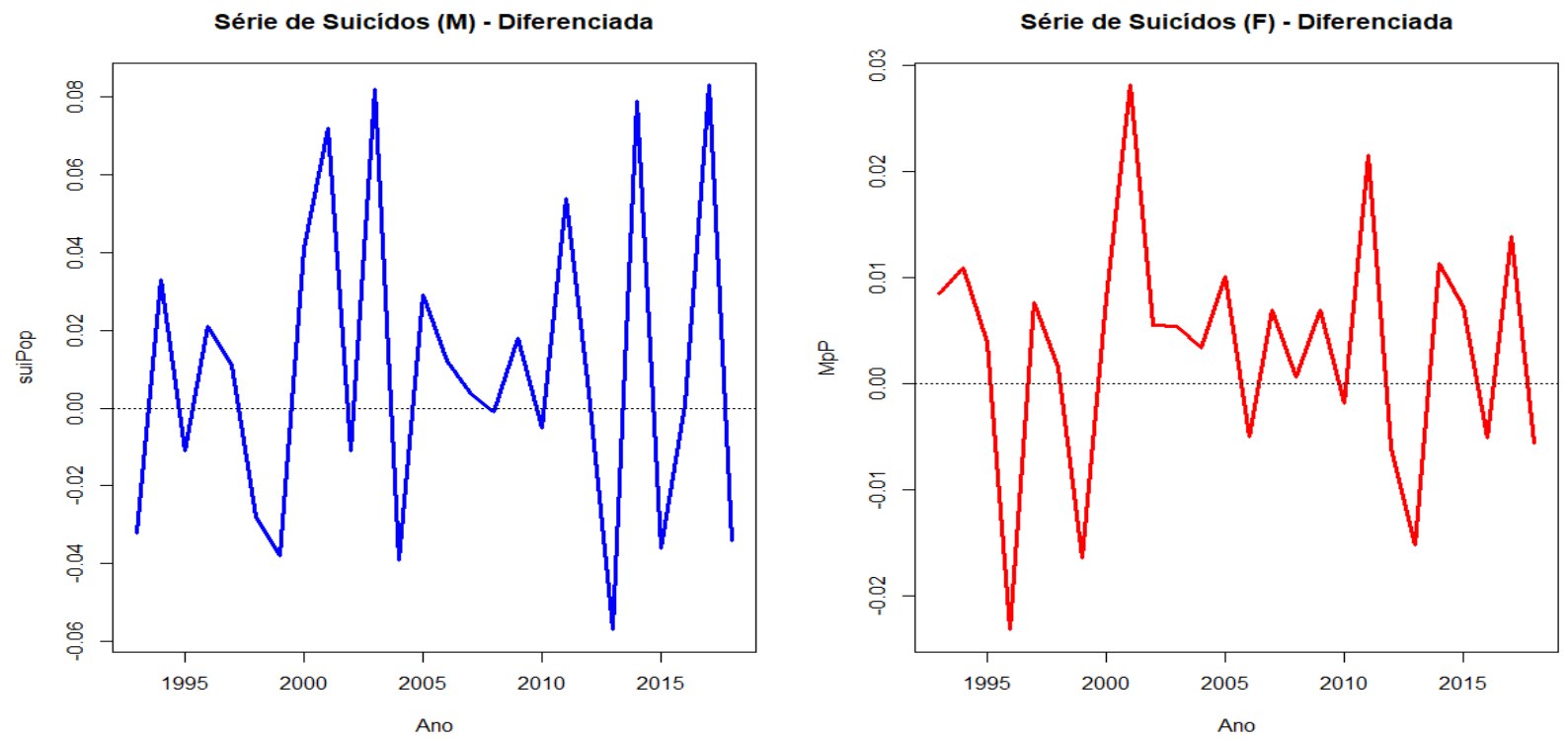

Figura 2 - Série diferenciada da taxa específica de suicídios em Minas Gerais

Para comprovar que após a primeira diferença as séries tornam-se estacionárias, foi aplicado novamente o teste $\mathrm{ADF}$ às séries diferenciadas. Com p-valores de 0,0044 para série feminina e 0,0010 para série masculina, comprova-se que após a primeira diferença as séries se tornaram-se estacionárias.

A próxima verificação realizada foi a presença ou não de sazonalidade; para isso foi aplicado o teste Kruskall Wallis, ao nível de 5\% de significância e com p-valores de 0,4631 
para série feminina e 0,4672 para a série masculina, temos evidências estatísticas de que as séries não possuem sazonalidade.

\subsection{Estimação dos modelos}

$\mathrm{Na}$ verificação da ordem do modelo a ser ajustado, foram analisados os gráficos das funções de autocorrelação e autocorrelação parcial das séries diferenciadas, que indicaram os primeiros modelos a serem experimentados. Na Figura 3 são mostradas as funções de autocorrelação e autocorrelação parcial das séries diferenciadas.
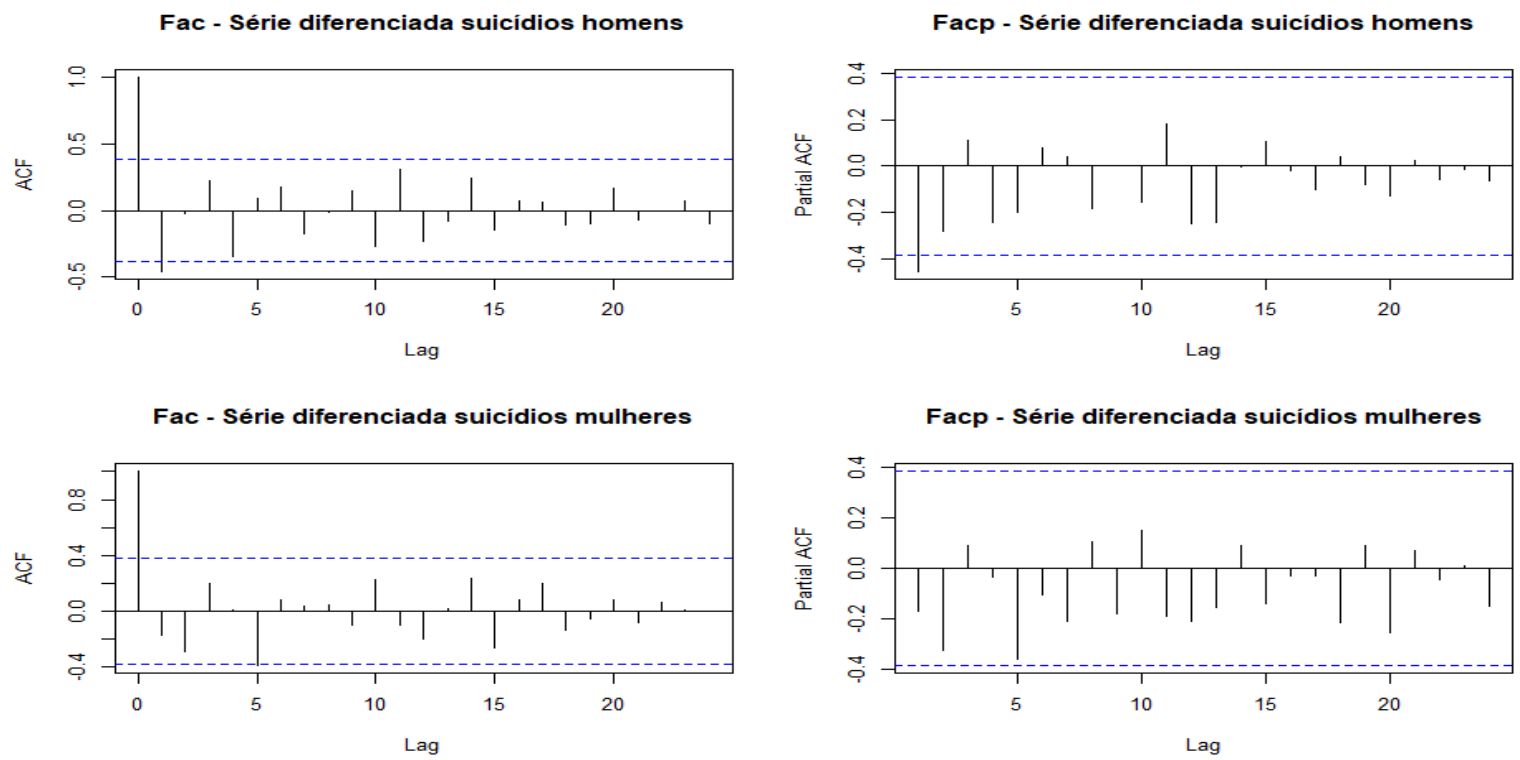

Figura 3 - Funções de autocorrelação e autocorrelação parcial das séries diferenciadas de Suicídios

Com as análises dos correlogramas na Figura 3, foram testados os modelos ARIMA $(1,1,2)$, ARIMA $(1,1,1)$ e ARIMA $(1,1,0)$ para a série masculina e ARIMA $(0,1,0)$, ARIMA $(0,1,1)$ e ARIMA $(0,1,5)$ para a série feminina. Como as séries tornaram-se estacionárias com a primeira diferença, todos os modelos foram rodados com $d=1$. Os critérios AIC para cada modelo são apresentados na Tabela 1. 
MARQUES, R. A.; SILVA, S. B. Análise temporal das taxas de suicídios no estado de Minas Gerais com modelos arima

Tabela 1 - Critério de Akaike para os modelos estimados

\begin{tabular}{llc}
\hline Sexo & \multicolumn{1}{c}{ Modelo } & AIC \\
\hline \multirow{3}{*}{ Masculino } & ARIMA(1,1,2) & $-59,39$ \\
& ARIMA $(1,1,1)$ & $-58,93$ \\
& ARIMA $(1,1,0)$ & $-57,04$ \\
\hline \multirow{3}{*}{ Feminino } & ARIMA $(0,1,0)$ & $-122,01$ \\
& ARIMA $(0,1,1)$ & $-121,62$ \\
& ARIMA $(0,1,5)$ & $-124,23$ \\
\hline \multicolumn{3}{c}{ Fonte: Autores }
\end{tabular}

Na Tabela 1, observa-se a partir do critério de AIC, que os melhores resultados foram os modelos ARIMA $(1,1,2)$ para a série masculina e ARIMA $(0,1,5)$ para a série feminina. Na Tabela 2, são mostrados os coeficientes, o erro padrão e o p-valor para cado modelo escolhido.

Tabela 2 - Parâmetros dos modelos ajustados

\begin{tabular}{lccc}
\hline Sexo & Modelo & Erro padrão & p-valor \\
\hline Masculino & $\phi_{1}=-0,8220$ & 0,1351 & 0,00021 \\
ARIMA $(1,1,2)$ & const $=0,0221$ & 0,1756 & 0,00059 \\
& $\theta_{2}=-1,0000$ & 0,2368 & 0,00002 \\
\hline Feminino & const $=0,0062$ & 0,0025 & 0,01550 \\
ARIMA $(0,1,5)$ & $\theta_{5}=-0,4269$ & 0,2135 & 0,04550 \\
\hline
\end{tabular}

Fonte: Autores

Os modelos para previsão podem ser escritos da seguinte forma:

$$
\hat{Z}_{t}=0,0221-0,8220 Z_{t-1}+a_{t-2}+a_{t}
$$

para o modelo ARIMA $(1,1,2)$ da taxa específica de suicídios da população masculina, e

$$
\hat{Z}_{t}=0,0062+0,4269 a_{t-5}+a_{t},
$$

para o modelo $\operatorname{ARIMA}(0,1,5)$ da taxa específica de suicídios da população feminina.

\subsection{Verificação dos modelos}

O próximo passo na análise de uma série temporal é verificar se o modelo ajusta-se de forma adequada aos dados, com um nível de significância pré estabelecido. Na figura 4 são mostrados os correlogramas dos resíduos para as séries de suicídios masculinos e femininos. Observa-se que os erros não são correlacionados, indicando um bom ajuste do modelo aos dados. 

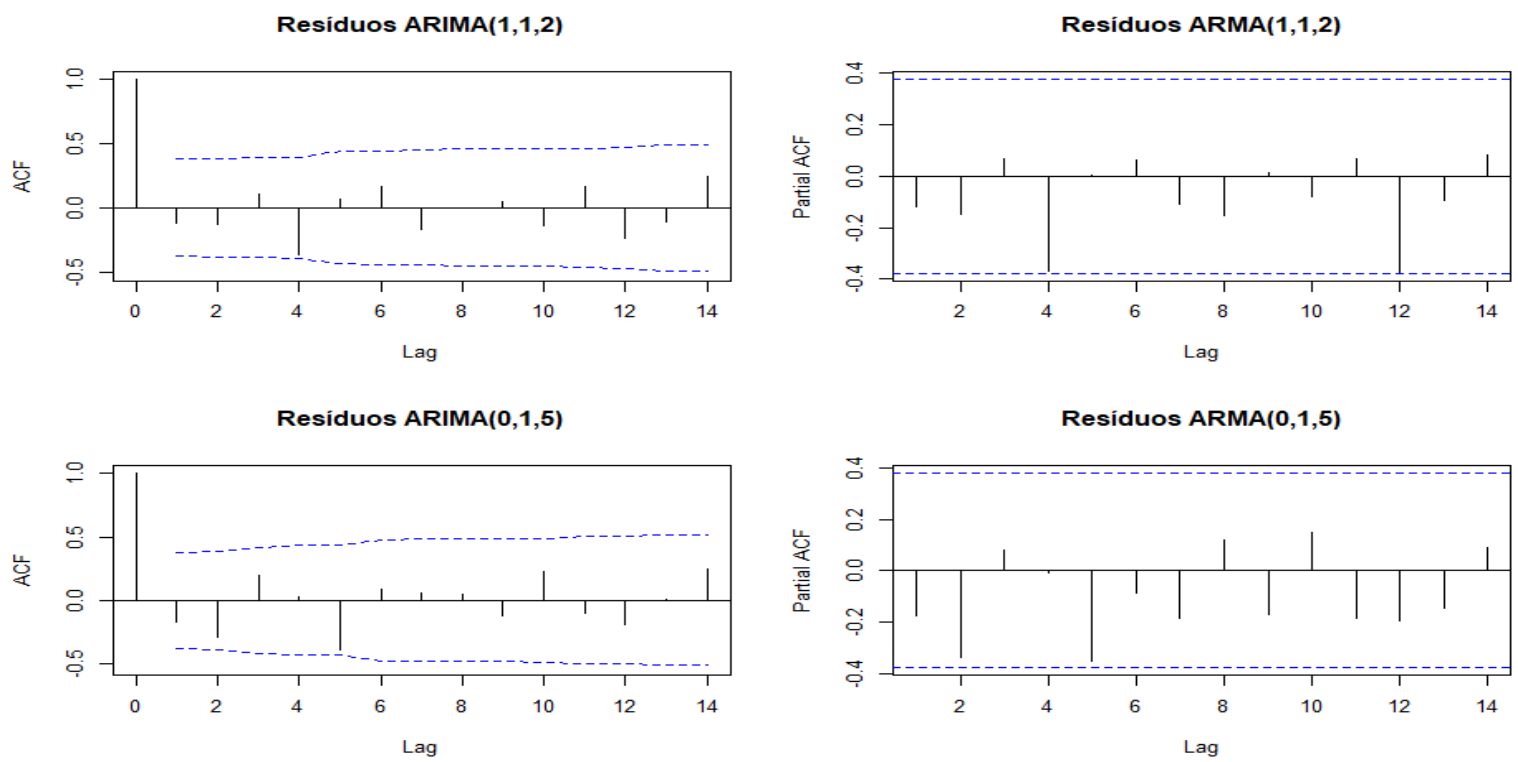

Figura 4 - Funções de autocorrelação e autocorrelação parcial dos resíduos

Considerando que o principal interesse no ajuste de séries temporais são valores futuros, então o modelo mais adequado é aquele que apresenta as melhores previsões. Foi aplicado o teste Ljung-Box e, com p-valores de 0,3770 para série feminina e 0,6153 para a série masculina, mostram-se que as séries ajustadas não apresentam correlação nos resíduos, comprovando o que já havia sido observado com as funções de autocorrelação na Figura 4.

\subsection{Previsões}

As séries em estudo apresentam valores da taxa específica de suicídios entre os anos de 1992 e 2018. Para verificar se os modelos apresentam previsões dentro do intervalo de confiança, foram retiradas as três últimas observações (2016, 2017 e 2018), ajustados os modelos propostos e feitas as previsões para essas observações retiradas. Na Tabela 3, são apresentadas as previsões para as duas séries por intervalo com $95 \%$ de confiança e os respectivos valores reais. 
MARQUES, R. A.; SILVA, S. B. Análise temporal das taxas de suicídios no estado de Minas Gerais com modelos arima

Tabela 3 - Previsões das séries retirando as últimas observações

\begin{tabular}{lccc}
\hline Série & Ano & Intervalo & valor real \\
\hline \multirow{3}{*}{ Prev (M) } & 2016 & $0,8646: 1,1575$ & 0.9863 \\
& 2017 & $0,8275: 1,1746$ & 1.1567 \\
& 2018 & $0,7978: 1,2115$ & 1.0836 \\
\hline \multirow{3}{*}{ Prev (F) } & 2016 & $0.2331: 0.3226$ & 0.2678 \\
& 2017 & $0.2146: 0.3411$ & 0.2950 \\
& 2018 & $0.2004: 0.3553$ & 0.2832 \\
\hline
\end{tabular}

Fonte: Autores

Nota-se que os valores das taxas específicas de suicídios reais estão dentro do intervalo de confiança previsto pelos modelos, sugerindo o bom ajuste dos modelos às séries analisadas. Após essa verificação, foram feitas previsões para os anos de 2019, 2020 e 2021 com os modelos propostos e com a série completa, ou seja, incluindo os valores reais de 2016, 2017 e 2018 . Na tabela 4, são apresentados os valores previstos para cada taxa específica e também por intervalo, ao nível de 5\% de significância.

Tabela 4 - Previsões futuras das séries

\begin{tabular}{lccc}
\hline Série & Ano & Intervalo & Previsões \\
\hline \multirow{3}{*}{ Prev (M) } & 2019 & $0,9900: 1,2285$ & 1,1092 \\
& 2020 & $1,0465: 1,2886$ & 1,1675 \\
& 2021 & $1,0409: 1,2856$ & 1,1632 \\
\hline \multirow{3}{*}{ Prev (F) } & 2019 & $0,2444: 0,3204$ & 0,2824 \\
& 2020 & $0,2319: 0,3394$ & 0,2856 \\
& 2021 & $0,2267: 0,3584$ & 0,2925 \\
\hline
\end{tabular}

Fonte: Autores

De acordo com a Tabela 4, observa-se que as previsões para a taxa de suicídios da população masculina apontam crescimento maior de 2019 para 2020, com crescimento médio anual de $5,1 \%$. As previsões de suicídios para população feminina também apontam um aumento nas taxas específicas para os próximos anos com crescimento médio anual de 2,3\%. Portanto, observando a tendência de ocorrências de suicídios para as duas populações, aspecto também observado por Ribeiro et al. (2018) na cidade de Uberaba em Minas Gerais, salienta-se a importância de políticas públicas serem direcionadas com mais atenção para essa temática, de forma a diminuir sofrimentos com a perda de vidas humanas. Na Figura 5, são mostradas graficamente essas conclusões. 
Previsões com modelo ARIMA(1,1,2) - Masculino

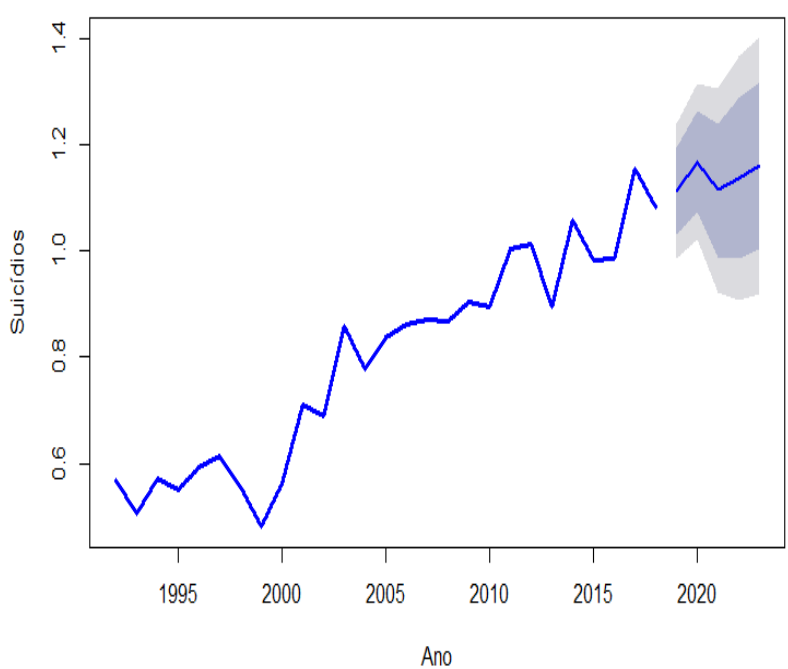

Previsões com modelo ARIMA $(0,1,5) \cdot$ Feminino

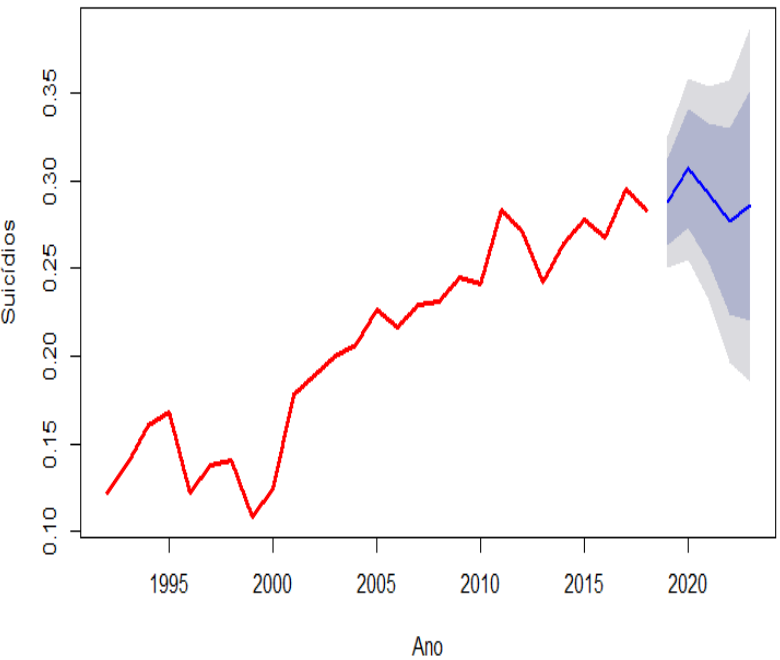

Figura 5 - Gráficos com previsões para as séries de suicídios por sexo em Minas Gerais

\section{CONCLUSÃO}

Esse estudo aplicou a metodologia Box et al. (1994) para ajustar modelos ARIMA às séries da taxa específicas de suicídios de homens e mulheres no estado de Minas Gerais entre os anos de 1992 e 2018 e, assim, obter previsões para os anos 2019, 2020 e 2021. Foi verificado, de maneira geral, que os modelos de Box \& Jenkins ajustaram-se bem aos dados a ponto de fazer previsões seguras com nível de significância de 5\%. Com isso, políticas públicas podem ser direcionadas com base nessas análises para salvar vidas. Foi observada uma tendência histórica de crescimento para as duas séries de suicídios e conclui-se que permanece a tendência de crescimento na taxa específica de suicídios para as duas populações (masculina e feminina) para os anos 2019, 2020 e 2021, caso nenhuma intervenção efetiva seja feita.

\section{REFERÊNCIAS}

ANDRADE, C. H. C. Manual de introdução ao pacote econométrico gretl. 2013. Disponível em: <https://s3.amazonaws.com/academia.edu.documents/39579087/Gretl.pdf.> Acesso em: 05 març. 2020.

ARAUJO, M. F. C. et al. Precipitação pluviométrica mensal no estado do rio de janeiro: sazonalidade e tendência. Bioscience Journal, Uberlândia, v. 25, n. 4, p. 90-100, 2009. 
BOX, G. E. et al. Time series analysis: forecasting and control. 3. ed. New Jersey: Prentice Hall, 1994.

BRANAS, C. C. et al. The impact of economic austerity and prosperity events on suicide in greece: a 30-year interrupted time-series analysis. BMJ open, British Medical Journal Publishing Group, v. 5, n. 1, 2015.

CAEIRO, F.; MATEUS, A. randtests: Testing randomness in R. [S.1.], 2014. R package version 1.0. Disponível em: <https://CRAN.R-project.org/package=randtests. $>$ Acesso em: 10 març. 2020.

CAMPOS, R. J. Previsão de séries temporais com aplicações a séries de consumo de energia elétrica. 2008. Disponível em: <https://repositorio.ufmg.br/bitstream/1843/ BUOS-8CTETD/1/290m.pdf>. Acesso em: 24 Març. 2020.

FILHO, M. C.; ZERBINI, T. Epidemiologia do suicídio no brasil entre os anos de 2000 e 2010. Saúde, Ética \& Justiça, v. 21, n. 2, p. 45-51, 2016.

GONÇALVES, L. R.; GONÇALVES, E.; JÚNIOR, L. B. d. O. Determinantes espaciais e socioeconômicos do suicídio no brasil: uma abordagem regional. Nova Economia, SciELO Brasil, v. 21, n. 2, p. 281-316, 2011.

GUJARATI, D. N.; PORTER, D. C. Econometria Básica-5. [S.1.]: Amgh Editora, 2011.

HYNDMAN, R. J.; KHANDAKAR, Y. Automatic time series forecasting: the forecast package for R. Journal of Statistical Software, [S.1.], v. 26, n. 3, p. 1-22, 2008. Disponível em: <http://www.jstatsoft.org/article/view/v027i03.> Acesso em: 10 març. 2020.

IPEA. Instituto de pesquisas econômicas e aplicadas. Tema: Saúde, 2020. Disponível em: <http://www.ipeadata.gov.br/Default.aspx.> Acesso em: 10 març. 2020.

KARAS, M.; URBANEK, J. runstats: Fast Computation of Running Statistics for Time Series. [S.1.], 2019. R package version 1.1.0. Disponível em: <https: //CRAN.R-project.org/package=runstats.> Acesso em: 10 març. 2020.

MCLEOD, A. I.; ZHANG, Y. Improved subset autoregression: With R package. Journal of Statistical Software, v. 28, n. 2, p. 1-28, 2008. Disponível em: <http: //www.jstatsoft.org/v28/i02/.> Acesso em: 10 març. 2020.

MORETTIN, P. A.; TOLOI, C. M. d. C. Análise de Séries Temporais. 2. ed. São Paulo: Edgard Blucher, 2006.

PFAFF, B. Analysis of Integrated and Cointegrated Time Series with R. 2. ed. New York: Springer, 2008. Disponível em: <http://www.pfaffikus.de.> Acesso em: 05 març. 2020.

RIBEIRO, N. M. et al. Análise da tendência temporal do suicídio e de sistemas de informações em saúde em relação às tentativas de suicídio. Texto \& Contexto-Enfermagem, SciELO Brasil, v. 27, n. 2, 2018. 
SILVEIRA, R. E.; SANTOS, S. A.; FERREIRA, L. A. Impacto da morbi-mortalidade e gastos com suicídio no brasil de 1998 a 2007. Revista de Pesquisa Cuidado é Fundamental Online, Rio de Janeiro, v. 4, n. 4, p. 3033-3042, 2012.

ZEILEIS, A.; HOTHORN, T. Diagnostic checking in regression relationships. R News, v. 2, n. 3, p. 7-10, 2002. Disponível em : <https://CRAN.R-project.org/doc/Rnews/.> Acesso em: 15 març. 2020.

\section{DADOS DOS AUTORES}

\section{Rodnei Alves Marques}

Lattes: http://lattes.cnpq.br/4173660058765943

E-mail: rodnei.marques@ifmg.edu.br

Licenciado em Matemática pela Universidade Federal de Minas Gerais (UFMG), Bacharel em estatística pela Universidade Federal de Ouro Preto (UFOP), Mestrado Profissional em Matemática pela Universidade Federal de Lavras (UFLA), curso de Formação Didática em Matemática pelo CIEP (Centre international d'études Pédagogiques - França), doutor em Estatística pela universidade Federal de Lavras (UFLA). Experiência na área de Matemática com ênfase em Álgebra e Cálculo, na área da Estatística com ênfase em Séries Temporais e Transformações Wavelets. Atualmente Professor do Instituto Federal de Minas Gerais - Campus Santa Luzia, com atuação nos cursos: Bacharelado em Engenharia Civil, Técnico em Edificações, Técnico em Segurança do Trabalho e Técnico em Paisagismo.

\section{Spencer Barbosa da Silva}

Lattes: http://lattes.cnpq.br/9371593871454464

E-mail: spencer.silva@ufop.edu.br

Possui graduação em Licenciatura Plena em Matemática pela Universidade Federal de Minas Gerais (2000), Especialização em Matemática (2002), Mestrado em Estatística pela Universidade Federal de Minas Gerais (2010) e doutorado em Estatística pela Universidade Federal de Minas Gerais (2015). Atualmente é professor Adjunto da Universidade Federal de Ouro Preto (UFOP). Tem experiência na área de Probabilidade e Estatística e Estatística Espacial, com ênfase em Detecção de clusters espaciais. 